\title{
Óleos essenciais uma alternativa para o controle de larvas do gênero Anopheles: uma
}

\section{revisão}

\author{
Essential oils an alternative for the control of genus Anopheles larvae: a review \\ Los aceites esenciales una alternativa para el control de las larvas de Anopheles: una revisión
}

Recebido: 12/08/2021 | Revisado: 17/08/2021 | Aceito: 18/08/2021 | Publicado: 22/08/2021

\author{
Amanda Leticia Fiorentino Mandu Bezerra \\ ORCID: https://orcid.org/0000-0002-6017-0736 \\ Universidade Federal Rural de Pernambuco, Brasil \\ E-mail: amandalebezerra01@gmail.com \\ Maria da Saúde da Silva \\ ORCID: https://orcid.org/0000-0002-1878-9690 \\ Universidade Federal Rural de Pernambuco, Brasil \\ E-mail: mariadasaudesilva8@gmail.com \\ Elayne Bessa Ferreira Pinheiro \\ ORCID: https://orcid.org/0000-0003-3696-8630 \\ Universidade Federal Rural de Pernambuco, Brasil \\ E-mail: elaynebessa@yahoo.com.br
}

\begin{abstract}
Resumo
Os óleos essenciais têm sido frequentemente utilizados como uma ferramenta alternativa para o controle de larvas e mosquitos de vários gêneros. O gênero Anopheles, conhecidos por ser o transmissor da malária, vem sendo testado com a utilização de óleos essenciais obtidos de diversas espécies de plantas. Esses mosquitos carregam como parasitas o protozoário do gênero Plasmodium que é o responsável por provocar a doença. Devido a necessidade de se conhecer cada vez mais alternativas para o controle desses vetores, o presente trabalho objetivou realizar um levantamento da utilização de óleos essenciais de diversas espécies no combate ao vetor da malária, ou seja, em mosquitos do gênero Anopheles, agindo diretamente nas larvas desses mosquitos. Esse levantamento foi realizado utilizando a base de dados Science Direct e Scielo, selecionando trabalhos dos anos de 2010 a 2020, analisando assim o título e objetivo dos mesmos. Nesse trabalho, foram avaliados os óleos essenciais de 33 espécies de plantas, pertencentes a 16 diferentes famílias contra as larvas de mosquitos desse gênero, sendo avaliada a eficácia do óleo essencial a partir do valor de $\mathrm{CL}_{50}$. Portanto, esse artigo destaca a grande relevância da utilização e eficácia de óleos essenciais no controle de mosquitos do gênero Anopheles.
\end{abstract}

Palavras-chave: Malária; Óleos essenciais; Atividade larvicida; Anopheles.

\begin{abstract}
Essential oils have often been used as an alternative tool for the control of larvae and mosquitoes of various genres. The genus Anopheles, known to be the transmitter of malaria, has been tested with the use of essential oils obtained from several plant species. These mosquitoes carry as parasites the protozoan of the genus Plasmodium, which is responsible for causing the disease. Due to the need to know more and more alternatives for the control of these vectors, the present study aimed to carry out a survey of the use of essential oils of different species to combat the malaria vector, that is, in mosquitoes of the genus Anopheles, acting directly on larvae of these mosquitoes. This survey was carried out using the Science Direct and Scielo database, selecting works from the years 2010 to 2020, thus analyzing their title and objective. In this work, the essential oils of 33 species of plants, belonging to 16 different families against mosquito larvae of this genus, were evaluated, and the effectiveness of the essential oil was evaluated from the value of $\mathrm{LC}_{50}$. Therefore, this article highlights the great relevance of the use and effectiveness of essential oils in the control of Anopheles mosquitoes.
\end{abstract}

Keywords: Malaria; Essential oils; Larvicidal activity; Anopheles.

\section{Resumen}

Los aceites esenciales se han utilizado a menudo como una herramienta alternativa para el control de larvas y mosquitos de varios géneros. El género Anopheles, conocido como transmisor de la malaria, ha sido probado utilizando aceites esenciales obtenidos de diferentes especies de plantas. Estos mosquitos portan como parásitos el protozoo del género Plasmodium, que es el responsable de provocar la enfermedad. Debido a la necesidad de conocer cada vez más alternativas para el control de estos vectores, este estudio tuvo como objetivo realizar un relevamiento del uso de aceites esenciales de diferentes especies en el combate al vector de la malaria, es decir, en mosquitos del género Anopheles. actuando directamente sobre las larvas de estos mosquitos. Esta encuesta se realizó utilizando las 
bases de datos Science Direct y Scielo, seleccionando trabajos de los años 2010 a 2020, analizando así su título y objetivo. En este trabajo se evaluaron los aceites esenciales de 33 especies vegetales pertenecientes a 16 familias diferentes frente a larvas de mosquito de este género, y se evaluó la efectividad del aceite esencial con base en el valor de $\mathrm{LC}_{50}$. Por ello, este artículo destaca la gran relevancia del uso y efectividad de los aceites esenciales en el control de los mosquitos Anopheles.

Palabras clave: Malaria; Aceites esenciales; Actividad larvicida; Anopheles.

\section{Introdução}

A malária é uma doença infecciosa febril aguda, cujos agentes etiológicos são protozoários transmitidos por vetores. Os mosquitos do gênero Anopheles são considerados os principais vetores de transmissão da malária, este grupo engloba cerca de 430 espécies, porém apenas aproximadamente 35 dessas, possuem comprovação de que são capazes de transmitir a malária (Schaffner et al.,2013).

De acordo com a Organização Mundial de Saúde (OMS) em 2015, os mosquitos anofelicos, principalmente o Anopheles gambie, são os principais causadores de doenças na África, sendo responsáveis por cerca de 200 milhões de casos de malária por ano no continente africano. Esses mosquitos carregam como parasitas o protozoário do gênero Plasmodium que é o responsável por provocar a doença. No Brasil, a presença de três tipos foram confirmados: o Plasmodium vivax, o falciparum e o malariae. Além deles, há o ovale e o knowlesi, que é um tipo mais raro. A contaminação, assim como ocorre com a Dengue, só acontece por meio da picada da fêmea do mosquito.

Somente em 2019, a OMS estimou a ocorrência de 228 milhões de casos de malária em todo mundo, sendo que países africanos e indianos possuíam cerca de $85 \%$ de todos esses casos e $95 \%$ de todas as mortes ocorridas pela doença, apresentando maior grau de mortalidade em crianças com idade menor que cinco anos.

No Brasil, entre o período de 2010 a 2019, foram registrados 7136 casos de malária onde a região Sudeste do país concentrou o maior número de casos em todo o período totalizando, somente nessa região, 3385 notificações (Rosa et al., 2020).

Os fatores socioeconômicos e ambientais como, por exemplo, a falta de instalações e drenagem adequada nas residências urbanas, a industrialização, o desmatamento, a construção de rodovias e hidrelétricas, dentre outros, tem provocado o aumento desses mosquitos transmissores (Gokhale et al., 2013). Com isso, tem se buscado várias alternativas para controle da doença, porém ela ainda afeta grande parte da população mundial. Na Tanzânia, por exemplo, em 2018 essa doença foi considerada a principal causa de mortalidade do país (Mboera et al., 2018).

Apesar dos grandes esforços mundiais para controle e combate a malária, esse ainda é um grande problema que ainda vem sendo diagnosticado em grande parte da população mundial e que o controle da transmissão dessa doença depende, principalmente, da distribuição dos vetores transmissores (Barik et al., 2015; Schaffner et al.,2013).

A redução da quantidade de mosquitos é o principal meio de controlar a grande taxa de transmissão da malária e para isso tem se utilizado diversas estratégias para o controle desses vetores de transmissão (Schaffner et al., 2013; Scott \& Morrison, 2010).

Para que ocorra o controle desses vetores têm sido utilizados inseticidas sintéticos, porém os mosquitos têm apresentado cada vez mais resistência a essas substâncias e esses compostos não são biodegradáveis (Kishore et al., 2011). Devido a isso, tem se buscado alternativas para encontrar outras formas de controle desses vetores como, por exemplo, a utilização de inseticidas naturais, que são biodegradáveis e apresentam mais segurança para o seu manuseio. Muitos desses produtos naturais tem se mostrado eficientes com atividades repelentes e larvicidas comprovadas para mosquitos do gênero (Pavela, 2015; Kishore et al., 2011). 
Com isso, o presente trabalho teve como objetivo realizar um levantamento bibliográfico da utilização de óleos essenciais de diversas espécies como uma alternativa no combate ao vetor da malária, ou seja, em mosquitos do gênero Anopheles, agindo diretamente nas larvas desses mosquitos.

\section{Metodologia}

Esse trabalho consiste em uma revisão bibliográfica a cerca da utilização dos óleos essenciais contra larvas pertencentes ao gênero Anopheles. Para sua produção foi realizado um levantamento de artigos científicos.

Os trabalhos foram adquiridos a partir da base de dados Science Direct e Scielo. A busca dos artigos foi realizada por meio de busca avançada nas bases de dados utilizando-se, como descritores controlados, os termos "essential oil and anopheles".

Dos artigos encontrados foram filtrados apenas os resultados dos anos 2010 a 2020. A seleção dos artigos foi realizada com base no título e no objetivo dos mesmos, sendo utilizados na construção desse trabalho apenas os artigos científicos que possuíam como objetivo a utilização de óleos essenciais no combate a larvas de mosquito do gênero Anopheles.

Esse estudo foi baseado em Estrela (2018) e Pereira (2018), possuindo características qualitativas a partir da análise documental dos artigos publicados na base de dados citado anteriormente.

\section{Resultados e Discussão}

Recentes investigações em vários países confirmam que alguns óleos essenciais de plantas não têm apenas a capacidade de repelir insetos, mas apresentam também ação inseticida através do contato direto ou pelas vias respiratórias dos insetos (Corrêa e Salgado 2011). Em 2005, a OMS publicou as Diretrizes para Laboratório e Ensaios de Campo quanto a atividade larvicida, com o objetivo de padronizar esses procedimentos para testar os mecanismos de ação do mesmo, no entanto, a OMS não estabeleceu um critério padrão para determinação da atividade larvicida de produtos naturais (OMS 2005), sendo assim, neste trabalho foi utilizado a classificação estabelecida por Cheng et al. (2003) em que as espécies testadas com $\mathrm{CL}_{50}>100 \mathrm{mg} / \mathrm{L}$ não exibiram um efeito larvicida, enquanto, aquelas com $\mathrm{CL}_{50}<100 \mathrm{mg} / \mathrm{L}$ apresentaram um significativo efeito larvicida.

Por meio da pesquisa realizada foram detectados 842 trabalhos, que foram filtrados, de acordo com o objetivo do trabalho, obtendo-se 34 espécies de plantas, pertencentes a 16 diferentes famílias, pelas quais foram testadas quanto à atividade larvicida contra mosquitos do gênero Anopheles. A Tabela 1 lista os óleos essenciais obtidos de distintas partes vegetais de diferentes espécies de plantas e seu efeito larvicida sobre diferentes espécies do mosquito do gênero Anopheles.

Nessa revisão foram detectadas seis espécies diferentes de mosquitos do gênero Anopheles como alvo de estudo, dentre essas espécies duas se destacam a Anopheles stephensi e a Anopheles gambiae representando 41,2 e 26,5\% dos trabalhos estudados, respectivamente. Anopheles stephensi é o principal mosquito vetor da malária nas áreas urbanas da Índia, essa espécie é uma das poucas espécies pertencentes ao gênero Anopheles que são encontradas em locais urbanos centrais. Presume-se que seja uma consequência direta de sua capacidade de localizar água limpa em tanques de armazenamento de água para colocar seus ovos (Sinka et al. 2011). Já Anopheles gambiae é um mosquito de origem africana, sendo o principal vetor da perigosa malária falcípara no continente africano (Gomes et al. 2011). No Brasil, ele foi introduzido no nordeste brasileiro, possivelmente, em 1930 vindo em barcos provenientes da costa africana, sendo o principal vetor de uma das maiores epidemias de malária registradas no Brasil (Lopes 2019).

Dentre as 16 famílias das espécies de plantas utilizadas nos estudos dos óleos essenciais como larvicida dos mosquitos do gênero Anopheles podemos destacar as famílias Lamiaceae, Asteraceae, Rutaceae e Zingiberaceae que foram responsáveis por $26,5,14,7,8,8$ e $8,8 \%$ das espécies estudadas, respectivamente. A família Lamiaceae também apresentou a maioria dos 
óleos ativos, 20,6\%, frente à atividade larvicida. Segundo alguns autores, as espécies de plantas pertencentes às famílias citadas anteriormente possui grande potencial para serem usados como bioinseticidas (Dias e Moraes 2013; Magalhães et al. 2010; Park et al. 2011).

As espécies de plantas que apresentaram os óleos essenciais mais ativos foram Rhizophora mucronata $\left(\mathrm{LC}_{50}=0,051\right.$ $\mathrm{mg} / \mathrm{L})$, Datura stramonium $\left(\mathrm{LC}_{50}=0,82 \mathrm{mg} / \mathrm{L}\right)$, Tagetes minuta $\left(L C_{50}=1,49 \mathrm{mg} / \mathrm{L}\right)$, Ocimum gratissimum $\left(\mathrm{LC}_{50}=2,35 \mathrm{mg} /\right.$ L) e Ocimum canum ( $\mathrm{LC}_{50}=3,38 \mathrm{mg} / \mathrm{L}$ ) (Afolabi et al. 2018, Kyarimpa et al. 2014, Yogananth et al. 2015), frente as larvas da espécie Anopheles stephensi para a Rhizophora mucronata e as larvas da espécie Anopheles gambiae para as demais. Com isso, é possível considerar os óleos dessas espécies de plantas como fontes promissoras ao combate desses mosquitos, sabe-se que o controle do mosquito na fase larval é uma prática eficaz para controlar as doenças transmitidas por mosquitos, pois a fase larval apresenta baixa mobilidade (Nandita el al. 2008).

Em 2015, Yogananth e seus colaboradores analisaram a composição química, atividade larvicida e repelente do óleo essencial de folhas da espécie Rhizophora mucronata (família Rhizophoraceae) contra duas espécies de mosquitos o Anopheles stephensi vetor da malária e o mosquito Culex quinquefasciatus vetor da filariose humana. Após a eclosão das larvas, essas foram expostas em contato com o óleo essencial da espécie da planta citada anteriormente e após $24 \mathrm{~h}$ foi observado a mortalidade das mesmas, apresentando $\mathrm{CL}_{50}$ de $0,051 \mathrm{mg} / \mathrm{L}$ para a espécie Anopheles stephensi, revelando, assim, que o óleo essencial possui atividade larvicida.

Segundo Simas et al. (2004), embora existam muitos relatos na literatura relatando atividade inseticida de óleos essenciais, poucos discutem a relação estrutura x atividade biológica de seus constituintes. Assim, observou-se que na análise dos ácidos graxos do óleo essencial ativo das folhas de Rhizophora mucronata, realizada por cromatografia gasosa acoplada à espectrometria de massa (CG-EM), foram identificados os compostos $\alpha$-pineno $(35,87 \%)$ e o ácido oleico $(33,87 \%)$ como constituintes majoritários e que esses compostos podem estar relacionados com a atividade larvicida do óleo essencial (Yogananth et al., 2015).

Segundo Simas et al. (2004) óleos essenciais ricos em monoterpenos, como o $\alpha$-pineno, são alternativas interessantes para o controle de larvas de mosquitos. Em uma busca por atividade larvicida observou-se para a resina de pinheiro (aguarrás), rica em $\alpha$-pineno e $\beta$-pineno, grande atividade larvicida frente ao Aedes aegypti. Os pinenos isolados foram testados e também apresentaram atividade larvicida (Lucia et al., 2007). Além disso, Gurunathan et al. (2016) sugeriram que o composto ácido oleico atua como um bom agente de controle de mosquitos, onde essa bioeficácia pode estar relacionada ao efeito decitinizante da parede corporal e a inibição da ecdisona 20 monooxigenase, uma enzima necessária para promover o desenvolvimento da membrana celular em insetos.

A composição do óleo essencial e a atividade larvicida utilizando larvas do mosquito Anopheles gambiae foram estudados por Kyarimpa et al. (2014) para a espécie Tagetes minuta (família Asteraceae). O óleo essencial da espécie foi obtido a partir do método de hidrodestilação e as larvas utilizadas nos ensaios pertenciam ao $3^{\circ}$ e $4^{\circ}$ estágio de desenvolvimento. A identificação dos compostos presentes no óleo essencial foi realizada por cromatografia gasosa acoplada a espectrometria de massa (CG-EM) onde indicaram a presença de trans-ocimeno (15,90\%), I-verbenona (15\%), limoneno (8,02\%) e 2-pinen-4-ona (7,84\%) como os principais compostos do óleo essencial. Após 12h da exposição das larvas ao óleo essencial, todas apresentaram mortalidade com $\mathrm{CL}_{50}$ de $1,49 \mathrm{mg} / \mathrm{L}$, sugerindo assim, que o óleo essencial de Tagetes minuta possui eficácia contra larvas. O mesmo ensaio realizado com o óleo essencial foi também realizado com o inseticida organofosforado metil paration, o qual apresentou resultados semelhantes aos do óleo essencial, o que reforça ainda que o resultado obtido com Tagetes minuta é promissor e que a espécie pode ser mais estudada para ser utilizada como uma alternativa na produção de inseticidas. 
Em 2018, Afolabi e colaboradores determinaram a eficácia adulticida e atividade repelente de óleos essenciais de diferentes espécies Ocimum canum, Ocimum gratissimum, Chromolaena odorata e Datura stramonium contra Anopheles gambiae adulto. Os extratos mostraram atividade adulticida em $\mathrm{LC}_{50}(0,82-4,52 \mathrm{mg} / \mathrm{L})$. A maior mortalidade foi observada em Datura stramonium e Ocimum gratissimum com valores de $\mathrm{LC}_{50}$ 0,82 $\mathrm{mg} / \mathrm{L}$ e $2,35 \mathrm{mg} / \mathrm{L}$. Enquanto isso, a menor atividade adulticida foi registrada em Chromolaena odorata com a CL50 mais baixa (4,52 mg/ L). Os resultados mostraram proteções completas de 90 min para Ocimum canum, 120 min para Ocimum gratissimum, 30 min para Chromolaena odorata e 120 min para Datura stramonium numa concentração de $5 \mathrm{mg} / \mathrm{L}$ dos óleos, respectivamente. Observou-se que D. stramonium e O. gratissimum proporcionaram maior proteção (120 min) contra Anopheles gambiae, enquanto a menor proteção (30 min) foi observada em Chromolaena odorata a $5 \mathrm{mg} / \mathrm{L}$. Os resultados do estudo mostraram que todos os óleos essenciais, com exceção de Chromolaena odorata, eram botânicos em potencial para confirmar a proteção nas partes expostas do corpo humano contra Anopheles gambiae por pelo menos 90 min e, como tal, podem ser incluídos no manejo integrado de vetores para reduzir infecção por malária em áreas endêmicas.

Ocimum gratissimum em seu óleo essencial destaca-se por apresentar como constituintes majoritários o eugenol, (Z)ß-ocimeno, germacreno D, (E)-cariofileno, geraniol e timol (Verma et al. 2013; Vieira et al. 2001; Vianna 2009; CaroviéStanko et al. 2011). Já os compostos linalool, neral, geranial, $\beta$-Caryophyllene, 1,8-Cineole e $\beta$-Pinene são constituintes majoritários do óleo essencial do Ocimum canum (Akgul et al. 1989; Bunrathep et al 2007; Zollo et al 1998).

Diversos estudos mostram que a atividade larvicida dessas duas espécies pode estar relacionada com a presença de dois compostos, como por exemplo, o eugenol e o timol associados aos seus demais constituintes (Barbosa et al. 2012; Barros Silva et al. 2017; Pandiyan et al. 2019; Yang et al. 2020).

De acordo com Yang et al. 2020 em seus estudos esses compostos apresentaram valores $\mathrm{LC}_{50}=19,8 \mathrm{mg} / \mathrm{L}_{\mathrm{e}} \mathrm{LC} \mathrm{C}_{50}=$ $37,1 \mathrm{mg} / \mathrm{L}$, respectivamente. Segundo Santos et al. (2011) a atividade larvicida de ambos os compostos permanece, fortemente, em sua passagem pela cutícula da larva, devido aos grupos hidrofóbicos de suas moléculas.

O efeito subletal dos óleos essenciais de diversas espécies de plantas mostra que não precisa apenas matar as larvas do mosquito para ser considerado eficaz no controle da malária, mas torná-las inativas ou limitar seu crescimento e que a fase não vetorial do ciclo de vida também é outra vantagem (Ayinde et al. 2020).

Os óleos essenciais possuem a capacidade de inibir o epitélio do intestino médio, o sistema respiratório, os cecos gástricos, os túbulos malphigian de larvas de mosquito e enfraquecer o sistema de defesa de uma larva, permitindo a fácil infiltração de organismos patogênicos no sistema de inseto (David et al. 2002; Rey et al. 1999; Su e Mulla, 1998a, b). Eles agem, também, inibindo a produção de ecdisona, uma enzima que permite a mudança da larva em mosquito, garantindo que a larva não atinja a fase adulta e permaneça no estágio larval e, em seguida, morra. Se, no entanto, a larva conseguir entrar na fase de pupa, existe a probabilidade de que permaneça absolutamente estéril, sem capacidade de reprodução (Ayinde et al. 2020).

Os óleos essenciais das espécies relatadas nesse trabalho (Tabela 1) além de poderem ser utilizados como uma alternativa ao combate aos mosquitos pertencentes ao gênero Anopheles, transmissores da malária, também podem atuar como substituintes dos inseticidas sintéticos. 
Tabela 1: Óleos essenciais obtidos de distintas partes vegetais e seu efeito larvicida sobre diferentes espécies do mosquito do gênero Anopheles, 2010 a 2020.

Família e espécies das

$\mathrm{LC}_{50}(\mathbf{p p m})$ plantas

Espécies de Anopheles

Referências

\section{ASTERACEAE}

Blumea martiniana

Tagetes minuta

Artemisia dracunculus

Chromolaena odorata

Annona senegalensis

\section{6,56}

1,49

11,36

4,52
Anopheles anthropophagus

Anopheles gambiae

Anopheles stephensi

Anopheles gambiae

Anopheles gambiae
Zhu \& Tian 2011

Kyarimpa et al. 2014

Osanloo et al. 2017

Afolabi et al. 2018

Younoussa et al. 2016

\begin{tabular}{|c|c|c|c|}
\hline APIACEA & & & \\
\hline Trachyspermum ammi & 43,84 & Anopheles stephensi & Vitali et al. 2016 \\
\hline Kelussia odoratissima & 4,88 & Anopheles stephensi & Vatandoost et al. 2012 \\
\hline \multicolumn{4}{|l|}{ BURSERACEAE } \\
\hline Boswellia dalzielii & - & Anopheles gambiae & Younoussa et al. 2016 \\
\hline \multicolumn{4}{|l|}{ MYRTACEAE } \\
\hline Eucalyptus camaldulensis & 397,75 & Anopheles stephensi & Medhi et al. 2010 \\
\hline \multicolumn{4}{|l|}{ MELIACEAE } \\
\hline Azadirachta indica & $-1666,86$ & Anopheles gambiae & Ayinde et al. 2020 \\
\hline \multicolumn{4}{|l|}{ RUTACEAE } \\
\hline Citrus sinensis & 77,55 & Anopheles labranchiae & El-Akhal et al. 2015 \\
\hline Citrus aurantium & 22,64 & Anopheles labranchiae & El-Akhal et al. 2015 \\
\hline Clausena anisata & 119,59 & Anopheles Stephensi & $\begin{array}{l}\text { Govindarajam et al. } \\
2010\end{array}$ \\
\hline \multicolumn{4}{|l|}{ SOLANACEAE } \\
\hline Datura stramonium & 0,82 & Anopheles gambiae & Afolabi, et al. 2018 \\
\hline \multicolumn{4}{|l|}{ CURCIBITACEAE } \\
\hline Coccinia indica & 16,5 & Anopheles stephensi. & Rajkumar et al. 2011 \\
\hline \multicolumn{4}{|l|}{ PIPERACEAE } \\
\hline Piper campense & 34,9 & Anopheles gambiae & Matasyoh et al. 2011 \\
\hline \multicolumn{4}{|l|}{ POACEAE } \\
\hline Cymbopogan citrates & 77,24 & Anopheles subpictus & Govindarajan 2011 \\
\hline \multicolumn{4}{|l|}{ LAURACEAE } \\
\hline Cinnamomum zeylanicum & 71,96 & Anopheles subpictus & Govindarajan 2011 \\
\hline Persea americana & 6,65 & Anopheles stephensi & Louis et al. 2020 \\
\hline \multicolumn{4}{|l|}{ LAMIACEAE } \\
\hline Rosmarinus officinalis & 64,50 & Anopheles subpictus & Govindarajan 2011 \\
\hline
\end{tabular}




\begin{tabular}{|c|c|c|c|}
\hline Ocimum basilicum & 9,75 & Anopheles subpictus & $\begin{array}{c}\text { Govindarajan et al. } \\
2013\end{array}$ \\
\hline Thymus transcaspicus & 134,1 & Anopheles stephensi & Dargahi et al. 2014 \\
\hline Nepeta menthoides & 234,3 & Anopheles stephensi & Mahnaz et al. 2012 \\
\hline Origanum vulgare & 21,15 & Anopheles stephensi & $\begin{array}{c}\text { Govindarajan et al. } \\
2016\end{array}$ \\
\hline Origanum vulgare & 24,06 & Anophels subpictus & $\begin{array}{c}\text { Govindarajan et al. } \\
2016\end{array}$ \\
\hline Coleus aromaticus & 22,32 & Anopheles stephensi & $\begin{array}{c}\text { Mathalaimuthu et al. } \\
2017\end{array}$ \\
\hline Ocimum canum & 3,38 & Anopheles gambiae & Afolabi, et al. 2018 \\
\hline Ocimum gratissimum & 2,35 & Anopheles gambiae & Afolabi, et al. 2018 \\
\hline \multicolumn{4}{|l|}{ ZINGIBERACEAE } \\
\hline Zingiber officinale & 57,98 & Anopheles subpictus & Govindarajan 2011 \\
\hline Hedychium larsenii & 10,45 e 14,68 & Anopheles stephensi & AlShebly et al. 2017 \\
\hline Amomum subulatum & 44,11 & Anopheles subpictus & $\begin{array}{l}\text { Govindarajan et al. } \\
2018\end{array}$ \\
\hline \multicolumn{4}{|l|}{ POLIGONACEAE } \\
\hline Polygonum hydropiper & 217 & Anopheles stephensi & $\begin{array}{l}\text { Maheswaran and } \\
\text { Ignacimuthu } 2013\end{array}$ \\
\hline \multicolumn{4}{|l|}{ CUPRESSACEAE } \\
\hline Juniperus procera & 14,42 & Anopheles arabiensi & $\begin{array}{l}\text { Karunamoorthi et al. } \\
\qquad 2014\end{array}$ \\
\hline \multicolumn{4}{|l|}{ RHIZOPHORACEAE } \\
\hline Rhizophora mucronata & 0,051 & Anopheles stephensi & Yogananth et al. 2015 \\
\hline
\end{tabular}

Fonte: Autores.

\section{Considerações Finais}

Mediante a busca por novas maneiras para realizar o controle de insetos aos quais são transmissores de diversas doenças, tem sido bastante aceito o uso de formulações de óleos essenciais como larvicidas eficazes devido à variedade de substâncias químicas orgânicas bioativas presentes neles, que possuem efeitos mais benéficos do que os inseticidas sintéticos e menos tóxicos para o meio ambiente.

A necessidade de incorporação de técnicas complementares para o controle integrado de vetores continua sendo a chave para reduzir a prevalência de doenças como, por exemplo, a malária. Como foi visto neste trabalho a importância dos óleos essenciais de origem vegetal são eficientes no controle de larvas do gênero Anopheles, tendo potencial para serem utilizados como biolarvicida No entanto, barreiras precisam, ainda, serem superadas para a comercialização de novos inseticidas botânicos.

Esperamos que este trabalho possa intensificar os estudos e, consequentemente, a elaboração de trabalhos acadêmicos que tenham foco na produção de novos agentes bioinseticidas para que, assim, possamos usufruir, de maneira significativa, de seus benefícios e, principalmente, possamos diminuir o número de pessoas que vem sendo atingidas, todos os anos, com doenças transmitidas por mosquitos.

\section{Agradecimentos}

Os autores agradecem a Universidade Federal Rural de Pernambuco (UFRPE) pelo apoio para a realização do trabalho. 


\section{Referências}

Afolabi, O. J., Simon-Oke, I. A., Elufisan, O. O. \& Oniya, M. O. (2018). Adulticidal and repellent activities of some botanical oils against malaria mosquito: Anopheles gambiae (Diptera: Culicidae).Beni-Suef University Journal of Basic and Applied Sciences, 7(1), 135-138. https://doi.org/10.1016/j.bjbas.2017.09.004

Akgul A. (1989). Volatile oil composition of sweet basil (Ocimum basilicum L.) cultivating in Turkey. Nahrung, 33, 87-88. https://doi.org/10.1002/food.19890330129

Alshebly, M. M., AlqahtaniL, F. S., Govindarajan, M., Gopinath, K., Vijayan, P. \& Benelli, G. (2016). Toxicity of ar-curcumene and epi- $\beta$-bisabolol from Hedychium larsenii (Zingiberaceae) essential oil on malaria, chikungunya and St. Louis encephalitis mosquito vectors. Ecotoxicology and environmental safety, 137, 149-157. 10.1016/ j.ecoenv.2016.11.028

Ayinde, A. A., Morakinyo, O. M. \& Sridhar, M. K. C. (2020). Repellency and larvicidal activities of Azadirachta indica seed oil on Anopheles gambiae in Nigeria. Heliyon, 6(5), e03920. 10.1016/j.heliyon.2020.e03920.

Barik, T. K. (2015) Antimalarial drug: from its development to deface. Current Drug Discovery Technologies, 12(4), 225-228. 10.2174 / 1570163812666150907100019 .

Bunrathep S., Palanuvez C. \& Ruangrungsi N. (2007). Chemical composition and antioxidative activities of essential oils from four Ocimum species endemic to Thailand. J Health Res., 21, 201-206. https://he01.tci-thaijo.org/index.php/jhealthres/article/view/154127/112179

Carovié-Stanko, K., Liber, Z., Politeo, O., Strikic, F., Kolak, I., Milos, M. \& Satovic, Z. (2011). Molecular and chemical characterization of the most widespread Ocimum species. Plant Systematics and Evolution, 294(3), 253-262. https://doi.org/10.1007/s00606-011-0471-X

Cheng, S. S., Chang, H. T., Chang, S. T., Tsai, K. H. \& Chen, W. J. (2003). Bioactivity of selected plant essential oils against the yellow fever mosquito Aedes aegypti larvae. Bioresour Technol. 89(1), 99-102. 10.1016 / s0960-8524 (03) 00008-7

Corrêa, J. C. R. \& Salgado, H. R. N. (2011). Atividade inseticida das plantas e aplicações: revisão. Revista Brasileira de Plantas Medicinais, 13(4), 500-506. http://dx.doi.org/10.1590/S1516-05722011000400016

David, J. P., Pautou, M. P. \& Meyran J. C. (2000). Differential Toxicity of leaf litter to dipteran larvae of mosquito development sites. J. Invertebr. Pathol, 75, 9-18. 10.1006/jipa.1999.4886

Dargahi, L., Razavi-Azarkhiavi, K., Ramezani, M., Abaee, M. R. \& Behravan, J. (2014). Insecticidal activity of the essential oil of Thymus transcaspicus against Anopheles stephensi. Asian Pacific Journal of Tropical Biomedicine, 4, 589-591. 10.12980/APJTB.4.2014APJTB-2014-0077

El-Akhal, F., Lalami, A. E. O. \& Guemmouh, R. (2015). Larvicidal activity of essential oils of Citrus sinensis and Citrus aurantium (Rutaceae) cultivated in Morocco against the malaria vector Anopheles labranchiae (Diptera: Culicidae). Asian Pacific Journal of Tropical Disease, 5(6), 458-462. 10.1016 / S22221808 (15) 60815-5

Estrela, C. (2018). Metodologia Científica: Ciência, Ensino, Pesquisa. (3a ed.), Editora Artes Médicas.

Gokhale, M. D., Paingankar, M. S. \& Dhaigude, S. D. (2013). Comparison of biological attributes of Culex quinquefasciatus (Diptera: Culicidae) populations from India. ISRN Entomology. https://doi.org/10.1155/2013/451592

Gomes, A. P., Vitorino, R. R., Costa, A. P., Mendonça, E. G., Oliveira, M. G. A. \& Siqueira-Batista, R. (2011). Malária grave por Plasmodium falciparum. Revista Brasileira de Terapia Intensiva, 23(3), 358-369. https://doi.org/10.1590/S0103-507X2011000300015

Govindarajan, M. (2010). Chemical composition and larvicidal activity of leaf essential oil from Clausena anisata (Willd.) Hook. f. ex Benth (Rutaceae) against three mosquito species. Asian Pacific Journal of Tropical Medicine, 3(11), 874-877. https://doi.org/10.1016/S1995-7645(10)60210-6

Govindarajan, M. (2011). Larvicidal and repellent properties of some essential oils against Culex tritaeniorhynchus Giles and Anopheles subpictus Grassi (Diptera: Culicidae). Asian Pacific Journal of Tropical Medicine, 4(2), 106-111. 10.1016/ S1995-7645 (11) 60047-3

Govindarajan, M., Rajeswary, M., Hoti, S. L. \& Benelli, G. (2016). Larvicidal potential of carvacrol and terpinen-4-ol from the essential oil of Origanum vulgare (Lamiaceae) against Anopheles stephensi, Anopheles subpictus, Culex quinquefasciatus and Culex tritaeniorhynchus (Diptera: Culicidae). Research in Veterinary Science, 104, 77-82. 10.1016/ j.rvsc.2015.11.011

Govindarajan, M., Sivakumar, R., Rajeswary, M. \& Yogalakshmi, K. (2013). Chemical composition and larvicidal activity of essential oil from Ocimum basilicum (L.) against Culex tritaeniorhynchus, Aedes albopictus and Anopheles subpictus (Diptera: Culicidae). Experimental parasitology, 134(1), 7-11. 10.1016/j.exppara.2013.01.018.

Govindarajan, M., Rajeswary, M., Senthilmurugan, S., Vijayan, P., Alharbi, N. S., Kadaikunnan, S. \& Benelli, G. (2018). Larvicidal activity of the essential oil from Amomum subulatum Roxb. (Zingiberaceae) against Anopheles subpictus, Aedes albopictus and Culex tritaeniorhynchus (Diptera: Culicidae), and non-target impact on four mosquito natural enemies. Physiological and Molecular Plant Pathology, 101, 219-224. https://doi.org/10.1016/j.pmpp.2017.01.003

Gurunathan, A., Senguttuvan, J. \& Paulsamy, S. (2016). Evaluation of mosquito repellent activity of isolated oleic acid, eicosyl ester from Thalictrum javanicum. Indian Journal of Pharmaceutical Sciences 78, 103-110. 10.4103 / 0250-474x.180259

Karunamoorthi, K., Girmay, A. \& Fekadu, S. (2014). Larvicidal efficacy of Ethiopian ethnomedicinal plant Juniperus procera essential oil against Afrotropical malaria vector Anopheles arabiensis (Diptera: Culicidae). Asian Pacific journal of tropical biomedicine, 4 , S99-S106. 10.12980 / APJTB.4.2014C687

Kishore, N., Mishra, B. B., Tiwari, V. K., Tripathi, V. \& Lall, N. (2014). Natural products as leads to potential mosquitocides. Phytochemistry Reviews, 13 (3), 587-627. https://doi.org/10.1007/s11101-013-9316-2 
Kyarimpa, C. M., Böhmdorfer, S., Wasswa, J., Kiremire, B. T., Ndiege, I. O. \& Kabasa, J. D. (2014). Essential oil and composition of Tagetes minuta from Uganda Larvicidal activity on Anopheles gambiae. Industrial Crops and Products, 62, 400-404. https://doi.org/10.1016/j.indcrop.2014.09.006

Lopes, G. (2019). Anopheles gambiae no Brasil: antecedentes para um "alastramento silencioso", 1930-1932. Hist. cienc. saude-Manguinhos, 26(3). https://doi.org/10.1590/S0104-59702019000300006

Louis, M. L. M., Pushpa, V., Balakrishna, K. \& Ganesan, P. (2020). Mosquito larvicidal activity of Avocado (Persea americana Mill.) unripe fruit peel methanolic extract against Aedes aegypti, Culex quinquefasciatus and Anopheles stephensi. South African Journal of Botany, 133, 1-4. https://doi.org/10.1016/j.sajb.2020.06.020

Lucia A, Gonzalez AP, Saccacini E, Licastro S, Zerba E. \& Masuh H. (2007). Larvicidal effect of Eucalyptus grandis essential oil and turpentine and their major components on A. Aegypti larvae. J Am Mosq Control Assoc, 23, 293-303. 10.2987 / 8756-971X (2007) 23 [299: LEOEGE] 2.0.CO; 2

Maheswaran, R. \& Ignacimuthu, S. (3013). Bioefficacy of essential oil from Polygonum hydropiper L. against mosquitoes, Anopheles stephensi and Culex quinquefasciatus. Ecotoxicology and environmental safety, 97, 26-31. 10.1016 / j.ecoenv.2013.06.028

Mahnaz, K., Alireza, F., Hassan, V., Mahdi, S., Reza, A. M. \& Abbas, H. (2012). Larvicidal activity of essential oil and methanol extract of Nepeta menthoides against malaria vector Anopheles stephensi. Asian Pacific Journal of Tropical Medicine, 5(12), 962-965. 10.1016/ S1995-7645 (12) 60182-5

Matasyoh, J. C., Wathuta, E. M., Kariuki, S. T. \& Chepkorir, R. (2011). Chemical composition and larvicidal activity of Piper capense essential oil against the malaria vector, Anopheles gambiae. Journal of Asia-Pacific Entomology, 14(1), 26-28. 10.1016/ j.aspen.2010.11.005

Mathalaimuth, B., Shanmugam, D., Kovendan, K., Kadarkarai, M., Jayapal, G. \& Benelli, G. (2017). Coleus aromaticus leaf extract fractions: a source of novel ovicides, larvicides and repellents against Anopheles, Aedes and Culex mosquito vectors? Process Safety and Environmental Protection, 106, 23-33. https://doi.org/10.1016/j.psep.2016.12.003

Mboera, L. E. G., Rumisha, S. F., Lyimo, E. P., Chiduo, M. G., Mangu, C. D., Mremi, I. R., Kumalija, C. J., Joachim, C., Kishamawe, C., Massawe, I. S., Matemba, L. E., Evord Kimario, E., Bwana, V. M. \& Mkwashapi, D. M. (2018). Cause-specific mortality patterns among hospital deaths in Tanzania, 20062015. PloS one, 13 (10), e0205833. https://doi.org/10.1371/journal. pone.0205833

Medhi, S. M., Reza, S., Mahnaz, K., Reza, A. M., Abbas, H., Fatemeh, M. \& Hassan, V. (2010). Phytochemistry and larvicidal activity of Eucalyptus camaldulensis against malaria vector, Anopheles stephensi.Asian Pacific Journal of Tropical Medicine, 3(11), 841-845. https://doi.org/10.1016/S19957645(10)60203-9

Nandita C., Subrata L. \& Goutam C. (2008). Mosquito larvicidal and antimicrobial activity of protein of Solanum villosum leaves. BMC Complement Altern Med., 8:62. 10.1186/1472-6882-8-62

OMS. (2020). WHO World Report on Malaria 2019 OMS.

OMS. (2015). WHO World Report on Malaria, OMS.

Osanloo, M., Amani, A., Sereshti, H., Abai, M. R., Esmaeili, F. \& Sedaghat, M. M. (2017). Preparation and optimization nanoemulsion of Tarragon (Artemisia dracunculus) essential oil as effective herbal larvicide against Anopheles stephensi. Industrial Crops and Products, 109, 214-219. https://doi.org/10.1016/j.indcrop.2017.08.037

Pavela, R. (2015). Essential oils for the development of eco-friendly mosquito larvicides: a review. Industrial crops and products, 76 , $174-187$. https://doi.org/10.1016/j.indcrop.2015.06.050

Pereira A. S. et al. (2018). Metodologia da pesquisa científica. UFSM

Rajkumar, S., Jebanesan, A. \& Nagarajan, R. (2011). Effect of leaf essential oil of Coccinia indica on egg hatchability and different larval instars of malarial mosquito Anopheles stephensi. Asian Pacific Journal of Tropical Medicine, 4(12), 948-951. 10.1016/ S1995-7645 (11) 60224-1

Rey D., Cuany A., Pautou M. \& Meyran J. (1999) Differential sensitivity of mosquito taxa to vegetable tannins. J. Chem. Ecol., 25, 537-548. 10.1023/A:1020953804114

Rosa, I. M. S., Trajano, I. L. O., Sá, A. F. C. M., Moura, L. V. M., Barros, M. C.; Marques Jr., D. V.; Fonseca, R. N. M. \& Marques, C. P. C. (2020). Epidemiologia da Malária no Brasil e resultados parasitológicos, de 2010 a 2019. Brazilian Journal of health Review, 3(5), 11484-11495. https://doi.org/10.34119/bjhrv3n5-010

Schaffner, F., Bellini, R., Petric, D., Scholte, E. J., Zeller, H. \& Rakotoarivony, L. M. (2013). Development of guidelines for the surveillance of invasive mosquitoes in Europe. Parasites \& vectors, 6(1), 209. https://doi.org/10.1186/1756-3305-6-209

Scott, T. W. \& Morrison, A. C. (2010). Longitudinal field studies will guide a paradigm shift in dengue prevention. In Vector biology, ecology and control, 139-161. Springer, Dordrecht. 10.1007 / 978-90-481-2458-9_10

Sinka, M. E., Bangs, M. J., Manguin, S. et al. (2011). The dominant Anopheles vectors of human malaria in the Asia-Pacific region: occurrence data, distribution maps and bionomic précis. Parasites Vectors, 4, 89. https://doi.org/10.1186/1756-3305-4-89

Simas, N. K.; Lima, E. C., Conceição, S. R. et al. (2004). Produtos naturais para o controle da transmissão da dengue: atividade larvicida de Myroxylon balsamum (óleo vermelho) e de terpenóides e fenilterpenóides. Química Nova, 27 (01), 46-49. https://doi.org/10.1590/S0100-40422004000100009

Su, T. \& Mulla, M. S. (1998). Antifeedancy of neem products containing Azadirachtin against Culex tarsalis and Culex quinquefasciatus (Diptera: Culicidae). J. Vector Ecol., 23 (2), 114-122. https://pubmed.ncbi.nlm.nih.gov/9879068/ 
Research, Society and Development, v. 10, n. 11, e37101119384, 2021

(CC BY 4.0) | ISSN 2525-3409 | DOI: http://dx.doi.org/10.33448/rsd-v10i11.19384

Su, T. \& Mulla, M. S. (1998). Ovicidal activity of neem products (azadirachtin) against Culex tarsalis and Culex quinquefasciatus (Diptera; Culicidae). J. Am. Mosq. Contr. Assoc., 14, 204-209. https://www.biodiversitylibrary.org/content/part/JAMCA/JAMCA_V14_N2_P204-209.pdf

Vatandoost, H., Dehkordi, A. S., Sadeghi, S. M. T., Davari, B., Karimian, F., Abai, M. R. \& Sedaghat, M. M. (2012). Identification of chemical constituents and larvicidal activity of Kelussia odoratissima Mozaffarian essential oil against two mosquito vectors Anopheles stephensi and Culex pipiens (Diptera: Culicidae). Experimental parasitology, 132(4), 470-474. 10.1016 / j.exppara.2012.09.010.

Verma, R. S., Padalia, R. C., Chauhan, A. \& Thul, S. T. (2013). Exploring compositional diversity in the essential oils of 34 Ocimum taxa from Indian flora. Industrial Crops and Products, 45, 7-19, 2013. 10.1016 / j.indcrop.2012.12.005

Vianna, J. S. (2009). Caracterização anatômica, morfológica e química de quimiotipos de Ocimum gratissimum Lineu. 78p. (Mestrado em Ciências Agrárias) Universidade de Brasília, Faculdade de Agronomia e Veterinária, Brasília. https://repositorio.unb.br/bitstream/10482/4469/3/2009_JulianaSantosVianna.pdf

Vieira, R. F., Grayer, R. J., Paton, A. \& Simon, J. E. (2001). Genetic diversity of Ocimum gratissimum L. based on volatile oil constituents, flavonoids and RAPD markers. Biochemical Systematics and Ecology, 29(3), 287-304. 10.1016 / s0305-1978 (00) 00062-4.

Vitali, L. A., Beghelli, D., Nya, P. C. B., Bistoni, O., Cappellacci, L., Damiano, S., Lupidi, G., Maggi, F., Orsomando, G., Papa, F., Petrelli, D., Petrelli, R., Quassinti, L., Majid, L. S., Zadeh, M. \& Bramucci, M. (2016). Diverse biological effects of the essential oil from Iranian Trachyspermum ammi. Arabian Journal of Chemistry, 9(6), 775-786. https://doi.org/10.1016/j.arabjc.2015.06.002

Yogananth, N., Anuradha, V., Ali, M. Y. S., Muthezhilan, R., Chanthuru, A. \& Prabu, M. M. (2015). Chemical properties of essential oil from Rhizophora mucronata mangrove leaf against malarial mosquito Anopheles stephensi and filarial mosquito Culex quinquefasciatus. Asian Pacific Journal of Tropical Disease, 5, 67-S72. https://doi.org/10.1016/S2222-1808(15)60859-3

Younoussa, L., Nukenine, E. N., Danga, S. P. Y. \& Esimone, C. O. (2016) Repellent activity of the creams formulated from Annona senegalensis and Boswellia dalzielii leaf fractions and essential oils against Anopheles gambiae (Diptera: Culicidae). Asian Pacific Journal of Tropical Disease, 6(12) 973-978. 10.1016 / S2222-1808 (16) 61167-2

Zhu, L. \& Tian, Y. J. (2011). Chemical composition and larvicidal effects of essential oil of Blumea martiniana against Anopheles anthropophagus. Asian Pacific Journal of Tropical Medicine, 4(5), 371-374. https://doi.org/10.1016/S1995-7645(11)60106-5

Zollo, P. H, Biyiti, L., Tchoumbougnang, F., Menut, C., Lamaty, C. \& Bouchet, P.H. (1998). Aromatic plants of tropical Africa. Part XXXII. Chemical composition and antifungal activity of thirteen essential oils from aromatic plants of Cameroon. Flavour Frag J., 13, 107-114. 10.1002/(SICI)1099$1026(199803 / 04)$ 